\title{
Ethanol-Herstellung mit Bakterien
}

\author{
Hermann Sahm und \\ Stephanie Bringer-Meyer*
}

\begin{abstract}
Zur Herstellung von Ethanol aus Zucker werden bislang Hefen, insbesondere Saccharomyces cerevisiae Stämme, verwendet. In den letzten Jahren konnte gezeigt werden, daß das Bakterium Zymomonas mobilis im Vergleich zur Hefe einige Vorteile für die Produktion von Industriealkohol hat. So ist die Ethanol-Produktionsgeschwindigkeit im Vergleich zu der herkömmlichen Hefegärung um den Faktor 5 bis 6 schneller. Ferner ist die Ethanol-Ausbeute um etwa $5 \%$ höher als bei Hefe, da bei diesem Bakterium weniger Zucker in das Zellmaterial eingebaut wird. Zymomonas mobilis hat - ähnlich wie die Hefe - eine außerordentlich hohe Ethanol-Toleranz, die es ermöglicht, aus entsprechend konzentrierten Zuckerlösungen Ethanol-Konzentrationen von über 13 Vol.- $\%$ zu erreichen. Untersuchungen des Lipidspektrums ergaben, daß dieses Bakterium große Mengen Hopanoide enthält, die vermutlich zur Stabilisierung der Zellmembranen gegenüber Ethanol von Bedeutung sind. Da für eine wirtschaftliche Ethanol-Produktion die Kosten für den Zucker von sehr großer Bedeutung sind, wurde die Vergärung einer Abfallstärke untersucht, die bei der Gewinnung von Glucosesirup aus Weizenmehl anfällt. Es konnte gezeigt werden, daß nach enzymatischer Verzuckerung dieser Abfallstärke die Glucose von Zymomonas mobilis sehr gut zu Ethanol vergoren werden kann. Mit Hilfe gentechnologischer Methoden soll das Substratspektrum von Zymomonas mobilis erweitert werden, so daB in Zukunft auch Pentosen wie Xylose oder Arabinose mit diesem Organismus zu Ethanol vergoren werden können.
\end{abstract}

Ethanol production with bacteria. Strains of Saccharomyces cerevisiae have mostly been used for the production of ethanol from sugar by yeasts. Recently it was shown that the bacterium Zymomonas mobilis has some advantages compared to yeast for the production of industrial alcohol. Compared to traditional yeast fermentation, ethanol yield is about $5 \%$ higher than with yeast, since less sugar is incorporated into cell material by this bacterium. Like yeast, Zymomonas mobilis has remarkably high ethanol tolerance which enables the bacterium to produce ethanol concentrations of more than 13 vol. $\%$ from sugar solutions of appropriate concentration. Investigations of the spectrum of lipids present have shown that this bacterium contains large quantities of hopanoids which are presumably of significance for the stabilization of cell membranes in the presence of ethanol. Since the cost of the sugar greatly influences the profitability fraction formed in the production of glucose syrup from wheat flour was investigated. It was shown that after enzymatic saccharification of this waste starch the glucose was efficiently fermented to ethanol by Zymomonas mobilis. It is planned to broaden the substrate spectrum of Zymomonas mobilis by gene cloning techniques so that in future pentoses, e.g. xylose or arabinose, can also be fermented to ethanol by this organism.
Die Gewinnung von alkoholischen Getränken wie Bier und Wein gehört wohl zu den ältesten von Menschen genutzten biotechnologischen Prozessen. Im Zweistromland stellten die Sumerer bereits vor 6000 Jahren das euphorisch belebende Getränk Bier her. Die babylonischen Exportbiere gelangten allmählich nach Ägypten, wo sich gewerbliche Brauereien mit gelernten Brauern entwickelten. Auch die Griechen und Römer kannten die Kunst des Bierbrauens, sie bevorzugten jedoch ein anderes biotechnologisches Produkt, den Wein. Auch wenn seit vielen tausend Jahren die Menschen alkoholische Getränke herstellen, so waren bis Mitte des letzten Jahrhunderts die wissenschaftlichen Erkenntnisse über die alkoholische Gärung sehr spärlich. Zwar konnte 1680 Antony van Leeuwenhoek, ein Kaufmann und Amateurwissenschaftler in Delft, mit einem selbstgebauten Mikroskop zum erstenmal Mikroorganismen sehen, unter anderem auch kleine rundliche Gebilde, vermutlich Hefen. Leeuwenhoek hatte jedoch keine Vorstellung von der Bedeutung dieser runden Gebilde und ahnte die Tragweite seiner Entdeckung nicht. Mitte des letzten Jahrhunderts wehrten sich die Chemiker Wöhler und Liebig noch gegen die Vorstellung, daß Hefen für die Umsetzung von Zucker zu Alkohol verantwortlich sein sollten. Erst Pasteur gelang es vor gut 100 Jahren nachzuweisen, daß die al-

\footnotetext{
* Prof. Dr. H. Sahm und Dr. S. Bringer-Meyer, Institut für Biotechnologie der Kernforschungsanlage Jülich GmbH, Postfach 1913, 5170 Jülich.
}

koholische Gärung bei der Bier- und Weinherstellung das Werk von Hefen ist. Die Hefen haben einen Durchmesser von etwa $5-10 \mu \mathrm{m}$ und vermehren sich durch Knospung. Bei ausreichender Sauerstoffversorgung (aeroben Bedingungen) können die Hefen den Zucker vollständig zu Kohlendioxid und Wasser abbauen und die dabei freiwerdende Energie für die Synthese von neuem Zellmaterial, Wachstum und Vermehrung, verwenden (Abb. 1). Unter Sauerstoffmangel (anaeroben Bedingungen) wird der Zucker in einer Art Disproportionierung zu Ethanol und $\mathrm{CO}_{2}$ umgesetzt, wobei nur relativ wenig Energie für die Hefezellen frei wird, so daß unter diesen Bedingungen nur ein geringes Wachstum möglich ist. Aus $100 \mathrm{~kg}$ Glukose können maximal $51 \mathrm{~kg}$ Ethanol und $49 \mathrm{~kg} \mathrm{CO}$, gebildet werden.

Während dieser Gärungsprozeß bisher hauptsächlich zur Erzeugung alkoholischer Getränke (Bier, Wein) eingesetzt wurde, hat der Gärungsalkohol in letzter Zeit auch als chemischer Rohstoff und als Energieträger Interesse gefunden. Dieser biologische Umwandlungsprozeß bietet die Möglichkeit, chemische Roh- und Brennstoffe aus den Zuckern der pflanzlichen Biomasse herzustellen, die mit Hilfe der Photosynthese aus Sonnenenergie, Kohlendioxid und Wasser ständig in groBen Mengen neu gebildet werden. Ethanol ist eine wichtige Industriechemikalie, von der im Jahre 1980 in der Bundesrepublik Deutschland 108000 t produziert wurden. Ethanol wird vorwiegend als Lösungs-, Extraktions- und Gefrierschutzmittel verwendet. Es dient darüber hinaus als Aus- 
(a)

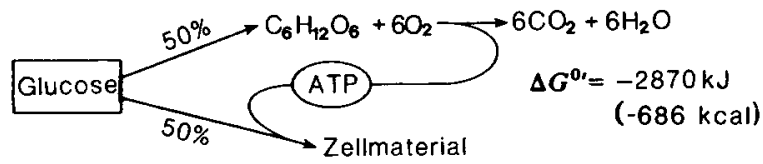

(b)

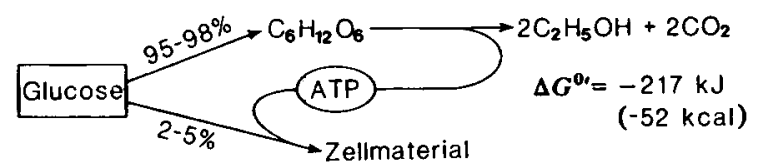

Abb. 1. Zuckerabbau durch Hefe unter a) aeroben und b) anaeroben Bedingungen.

gangssubstanz für die Synthese anderer organischer Verbindungen, die als Lösungsmittel, Extraktionsmittel, Farbstoffe, Klebstoffe, Schmiermittel, Pharmazeutika, Detergentien, Pestizide, Weichmacher, Mittel zur Oberflächenveredlung, Kosmetika und Kunststoffharze Verwendung finden. Ethanol wird entweder synthetisch aus petrochemischen Grundstoffen (Ethylen) oder biologisch aus Zucker mit Hilfe von Mikroorganismen gewonnen.

Obwohl viele Mikroorganismen in der Lage sind unter anaeroben Bedingungen Glukose zu Ethanol zu vergären, werden zur industriellen Herstellung von Ethanol bis heute ausschließlich Hefen verwendet. In großen Gärbehältern (50-1000 $\left.\mathrm{m}^{3}\right)$ wird die auf $\mathrm{pH} 4-5$ eingestellte Zuckerlösung mit einer Hefesuspension beimpft. Während der Wachstumsphase bei $30^{\circ} \mathrm{C}$ wird dann die Glukose zu Ethanol und Kohlendioxid vergoren. Nach etwa 2-3 Tagen ist die Gärung abgeschlossen, und die Ausbeute an Ethanol liegt bei etwa 85-90\% bezogen auf den theoretischen maximal erreichbaren Wert. Durch Destillation und Rektifikation wird dann das Ethanol aus dem Kulturmedium gewonnen, der Rückstand, die Schlempe, kann als Viehfutter verwendet werden. Die hohen Ölpreise der 70iger Jahre haben dazu geführt, daß durch Gärung gewonnener Alkohol als Benzinzusatz in manchen Ländern sehr großes Interesse gefunden hat. So produzierte Brasilien 1982 5,2 Millionen $\mathrm{m}^{3}$ Ethanol aus Zuckerrohr; bis zu den 90er Jahren plant Brasilien, Benzin vollständig durch Alkohol zu ersetzen. Im Mittelwesten der USA hat Gasohol, eine $9+1$ Mischung aus Benzin und Ethanol, großes Interesse gefunden.

\section{Ethanol-bildende Bakterien}

Wie aus Tab. 1 zu ersehen ist, haben in letzter Zeit eine Reihe von fakultativ anaeroben und obligat anaeroben Bakterien für die Ethanol-Produktion großes Interesse gefunden [1].

So bieten thermophile Bakterien, die ihr Wachstumsoptimum zwischen 60 und $70^{\circ} \mathrm{C}$ haben, im Vergleich zur Hefegärung folgende Vorteile:

- Die Kühlkosten für die Abführung der entstehenden Prozeßwärme sind geringer.

- Da die Viskosität des Kulturmediums mit steigender Temperatur abnimmt, ist der Bedarf an Energie für die Rührung des Mediums geringer.

- Das gebildete Ethanol kann schon durch Anlegen eines geringen Vakuums kontinuierlich aus dem Kulturmedium abgetrennt werden.

Unter den mesophilen Bakterien scheint besonders Zymomonas mobilis, ein Organismus, der Anfang dieses Jahrhunderts aus Palmwein und dem mexikanischen Getränk Pulque isoliert wurde, für die Ethanol-Produktion geeignet zu sein [2, 3]. Im Vergleich zur Hefe hat dieses Bakterium folgende Vorteile (s. auch Tab. 2):
Tabelle 1.

Bakterien, die in jüngster Zeit Interesse für die Ethanol-Produktion gefunden haben.

\begin{tabular}{|c|c|c|c|c|}
\hline Organismen & $\begin{array}{l}\text { Wachstums- } \\
\text { temperatur }\end{array}$ & $\begin{array}{l}\text { Hexose- } \\
\text { Vergärung }\end{array}$ & $\begin{array}{l}\text { Pentose- } \\
\text { Vergärung }\end{array}$ & Literatur \\
\hline \multicolumn{5}{|l|}{ Fakultativ anaerob: } \\
\hline Bacillus macerans & 37 & + & + & [12] \\
\hline $\begin{array}{l}\text { Klebsiella plan- } \\
\text { ticola }\end{array}$ & 30 & + & + & {$[15]$} \\
\hline \multicolumn{5}{|l|}{ Obligat anaerob: } \\
\hline $\begin{array}{l}\text { Zymomonas } \\
\text { mobilis }\end{array}$ & 30 & + & - & {$[4]$} \\
\hline Sarcina ventriculi & 37 & + & $+1)$ & {$[16,17]$} \\
\hline \multicolumn{5}{|l|}{ Clostridium } \\
\hline saccharolyticum & 37 & + & + & {$[18]$} \\
\hline $\begin{array}{l}\text { thermocellum } \\
\text { thermosac- }\end{array}$ & $55-62$ & + & + & [19] \\
\hline $\begin{array}{l}\text { charolyticum } \\
\text { thermohy- }\end{array}$ & $58-64$ & + & + & [19] \\
\hline drosulfuricum & 69 & + & + & [19] \\
\hline \multicolumn{5}{|l|}{$\begin{array}{l}\text { Thermoanaero- } \\
\text { bacter }\end{array}$} \\
\hline ethanolicus & 69 & + & + & [19] \\
\hline finnii & $64-66$ & + & + & {$[20]$} \\
\hline brockii & $65-70$ & + & $+1)$ & [19] \\
\hline
\end{tabular}

1) nur Arabinose

- Die Wachstumsgeschwindigkeit ist etwa doppelt so hoch wie bei der Hefe.

- Die Ethanol-Produktionsgeschwindigkeit ist im Vergleich zur herkömmlichen Hefegärung um den Faktor 6 bis 7 schneller. Dies ist sehr wahrscheinlich darauf zurückzuführen, daß bei diesem kleinen Bakterium (Größe 1 bis $2 \mu \mathrm{m}$ ) der Stofftransport besser erfolgen kann, als bei der Hefezelle (Größe 5 bis $10 \mu \mathrm{m}$ ).

- Die Ethanol-Ausbeute ist um etwa $5 \%$ höher als bei der Hefe, da bei diesem Bakterium weniger Zucker im Zellmaterial eingebaut wird.

Wie stoffwechselphysiologische Untersuchungen ergeben haben, baut das Bakterium Zymomonas mobilis den Zucker nicht über den Fructose-1,6-diphosphat-Weg wie die Hefe sondern über den 2-Keto-3-desoxy-6-phosphogluconat-Weg ab [4]. Wie aus Abb. 2 zu ersehen ist, wird auch hierbei als Zwischenprodukt Pyruvat gebildet, das wie in den Hefen zu Acetaldehyd decarboxyliert und anschließend zu Ethanol reduziert wird, so daß pro mol Glukose 2 mol Ethanol und 2 mol $\mathrm{CO}_{2}$ entstehen. Im Gegensatz zur Hefe ist jedoch die Energie(ATP)-Ausbeute, bezogen auf die umgesetzte Glukose, bei diesem Bakterium nur halb so groß. Dies hat einerseits zur Folge, daß Zymomonas mobilis aufgrund dieses geringen Energiegewinns nur halb so viel Zellmasse pro mol umgesetz-

Tabelle 2.

Gärungseigenschaften des Bakteriums Zymomonas mobilis und der Hefe Saccharomyces carlsbergensis.

\begin{tabular}{lcc}
\hline & $\begin{array}{c}\text { Zymomonas } \\
\text { mobilis }\end{array}$ & $\begin{array}{c}\text { Saccharomyces } \\
\text { carlsbergensis }\end{array}$ \\
\hline $\begin{array}{l}\text { Verdopplungszeit }[\mathrm{h}] \\
\text { Ethanol-Produktionsgeschwindigkeit } \\
\quad q_{\mathrm{p} / \mathrm{x}}\left[\mathrm{g} \mathrm{g}^{-1} \mathrm{~h}^{1}\right]\end{array}$ & 2,51 & 5,64 \\
$\begin{array}{l}\text { Zellausbeute } \\
\quad Y_{\mathrm{x} / \mathrm{s}}\left[\mathrm{g} \mathrm{g}^{-1}\right]\end{array}$ & 5,44 & 0,82 \\
$\begin{array}{l}\text { Produktausbeute } \\
Y_{\mathrm{p} / \mathrm{s}}[\%]\left(100 \%=0,511\left[\mathrm{~g} \mathrm{~g}^{-1}\right]\right)\end{array}$ & 0,028 & 0,043 \\
\hline
\end{tabular}




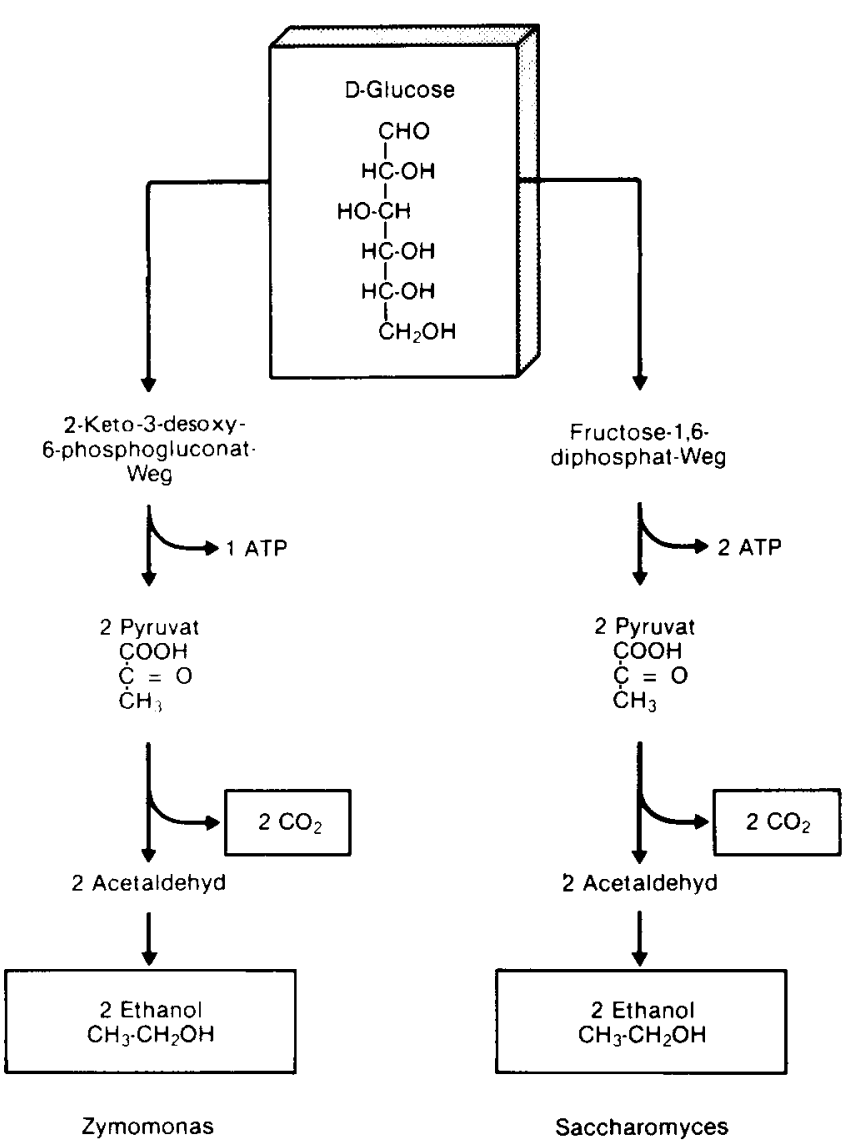

Abb. 2. Stoffwechselwege für die Vergärung von Glucose zu Ethanol in Zymomonas mobilis und in der Hefe Saccharomyces cerevisiae.

te Glukose neu synthetisieren kann als die Hefe, was andererseits zu einer höheren Ethanol-Ausbeute bei diesem Bakterium führt.

Wie bereits erwähnt, bildet die Hefe Saccharomyces cerevisiae nur dann aus der Glukose in hoher Ausbeute Ethanol, wenn kein Sauerstoff zur Veratmung des Zuckers zu $\mathrm{CO}_{2}$ und Wasser zur Verfügung steht. Andererseits benötigt die Hefe jedoch zur Synthese einiger lebenswichtiger Zellbestandteile (z. B. Steroide, ungesättigte Fettsäuren) etwas Sauerstoff. Es ist deshalb bei der Ethanol-Fermentation notwendig, die Sauerstoffversorgung so einzustellen, daß sie einerseits für das Wachstum der Hefezellen noch ausreicht, aber andererseits nicht zuviel Zucker zu $\mathrm{CO}$, und Wasser veratmet werden kann. Im Gegensatz dazu konnte gezeigt werden, daß das Bakterium Zymomonas mobilis für das Wachstum keinen Sauerstoff benötigt (Abb. 3), so daß mit diesem Organismus die Prozeßführung bei der Ethanol-Produktion einfacher ist. Im Vergleich zu einer Reihe strikt anaerober Bakterien ist Zymomonas mobilis relativ unempfindlich gegenüber Sauerstoff und besitzt ein Elektronen-Transportsystem, das der Atmungskette von aeroben Organismen ähnlich ist [5]. Dieses Bakterium kann somit Wasserstoff, der bei der Umsetzung von Glukose zu Pyruvat entsteht, auf molekularen Sauerstoff übertragen; da dann jedoch die Redoxbilanz bei der Glukosevergärung zu Ethanol nicht mehr ausgeglichen ist, kommt es unter diesen Bedingungen zur Bildung von Acetaldehyd, der einen wachstumshemmenden Effekt auf diesen Mikroorganismus ausübt [6] (Abb. 4).

\section{Ethanol-Toleranz}

Während die meisten Bakterien durch eine Ethanol-Konzentration von 10 bis $20 \mathrm{~g} / \mathrm{l} \mathrm{im}$ Wachstum gehemmt werden, hat Zymomonas mobilis, ähnlich wie die Hefe, eine außerordentlich hohe Ethanol-Toleranz, die es ermöglicht aus entsprechend konzentrierten Zuckerlösungen Alkoholkonzentrationen von $120 \mathrm{~g} / \mathrm{l} \mathrm{zu}$ erreichen [2]. Wie eine Reihe von Untersuchungen gezeigt haben, führen hohe Ethanol-Konzentrationen zur Zerstörung der Struktur und Funktion der Zellmembranen [7]. Kürzlich konnten in Zymomonas mobilis große Mengen pentacyclischer Triterpenoide (Hopanoide) nachgewiesen werden, die vor einigen Jahren zum erstenmal in verschiedenen Erdölfraktionen entdeckt wurden [8, 9] (Abb. 5). Der Gehalt an Tetrahydroxybakteriohopan $(\mathrm{THBH})$ in den Bakterienzellen nimmt mit zunehmender Ethanol-Konzentration sehr stark zu (Abb. 6), was darauf hinweist, daß es sehr wahrscheinlich einen Zusammenhang zwischen dem Hopanoid-Gehalt und der Ethanol-Toleranz gibt. Der durch Ethanol hervorgerufenen Fluiditätsänderung der Membran scheint Zymomonas mobilis durch den vermehrten Einbau von Hopanoiden entgegenwirken zu können. Vermut-
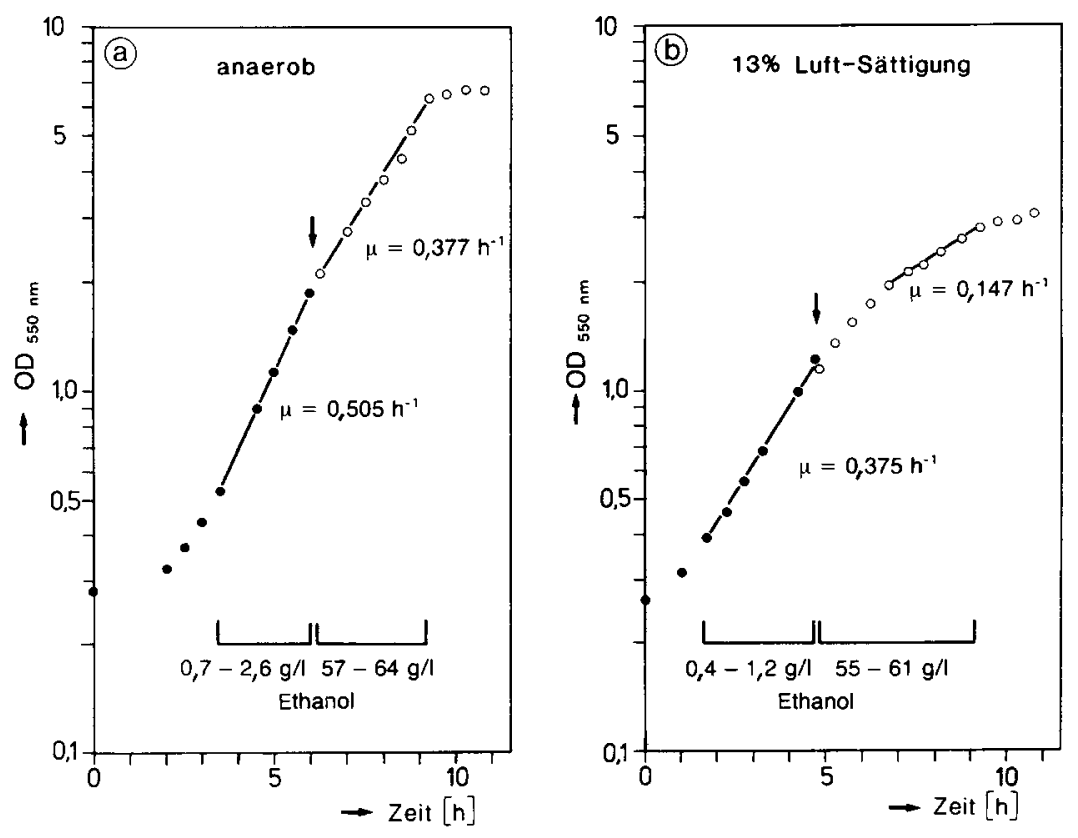

Abb. 3. Einfluß von Sauerstoff auf die Wachstumsrate $\mu\left[\mathrm{h}^{-1}\right]$ von Zymomonas mobilis bei verschiedenen Ethanol-Konzentrationen. a) Anaerobe Kultur; b) Kultur bei ca. $13 \%$ Luftsauerstoff-Sättigung. 


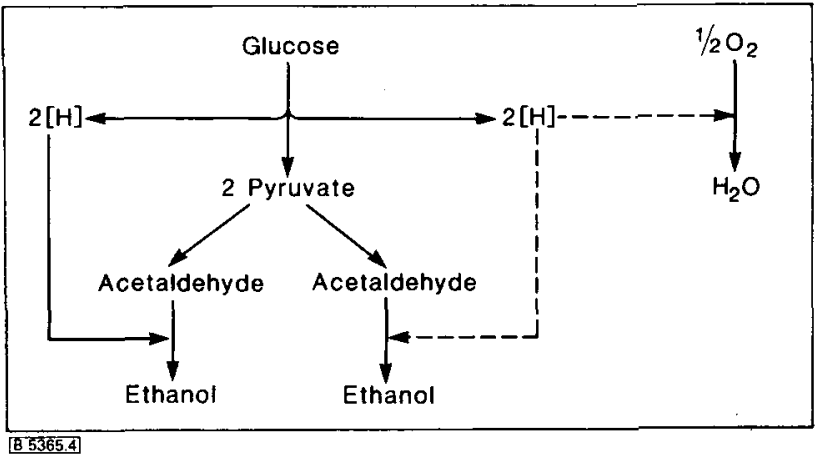

Abb. 4. Einfluß von Sauerstoff auf die Redoxbilanz bei der Glucosevergärung durch Zymomonas mobilis.

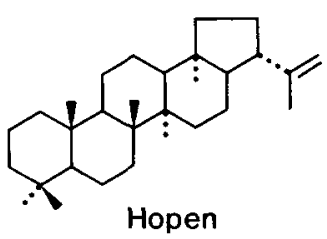

(Hop-22(29)-en Diplopten)

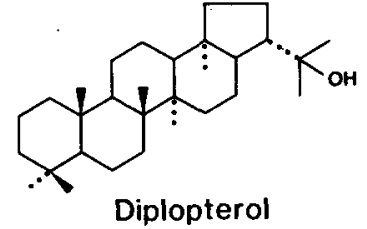

(Hopan-22-01)

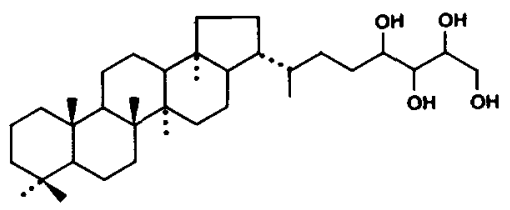

Tetrahydroxybakteriohopan "Tetrol"

$8 \overline{5365.5}$

Abb. 5. Hopanoide aus Zymomonas mobilis.

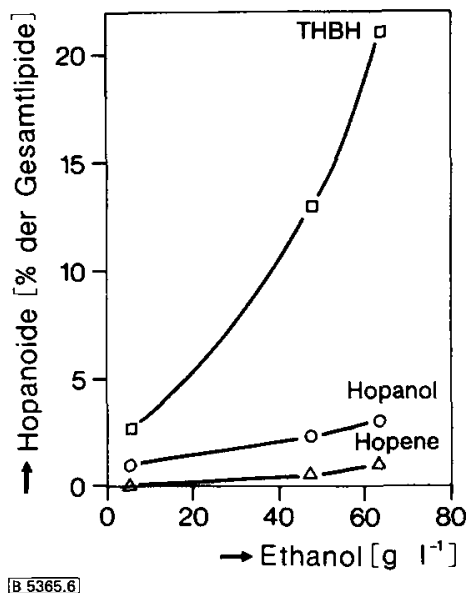

Abb. 6. Hopanoid-Gehalte in Zymomonas-Zellen in Abhängigkeit von der Ethanol-Konzentration.

lich haben die Hopanoide bei den Bakterien eine ähnliche Funktion, wie die Steroide bei Hefen. Es sind Membranstabilisatoren, die für die Ethanol-Toleranz von großer Bedeutung sind [10]. Während jedoch für die Steroid-Biosynthese Sauerstoff notwendig ist, erfolgt die Synthese der Hopanoide in den Bakterien auch unter anaeroben Bedingungen. Laufende Untersuchungen zur Aufklärung der Regulation der HopanoidBiosynthese in diesen Bakterien sollen mittelfristig dazu beitragen, die Ethanol-Toleranz in Bakterien gezielt verändern zu können.

\section{Kontinuierliche Ethanol-Fermentation}

Während bislang bei den meisten biotechnologischen Verfahren statische oder Batch-Kulturen verwendet werden, gewinnen in jüngster Zeit die kontinuierlichen Prozesse zunehmend an Bedeutung. In einer kontinuierlichen Kultur ist es möglich, die Zellen über lange Zeit bei gleichbleibender Substratkonzentration und konstanten Kulturbedingungen in der exponentiellen Wachstumsphase zu halten. Auf diese Weise kann die Produktivität im Vergleich zu den Batch-Kulturen ganz erheblich gesteigert werden. Während die Ethanol-Fermentation mit Hefen bis heute vorwiegend in Batch-Kulturen erfolgt, konnte mit dem Bakterium Zymomonas mobilis in einer kontinuierlichen Kultur bei einer Verdünnungsrate von 0,07 $\mathrm{h}^{-1}$ eine Zuckerlösung von $135 \mathrm{~g} / \mathrm{l}$ nahezu quantitativ zu Ethanol über einen Zeitraum von mehr als $900 \mathrm{~h}$ vergoren werden [11] (Abb. 7). Die stationäre Ethanol-Konzentration lag dabei zwischen 60 und $65 \mathrm{~g} / \mathrm{l}$.

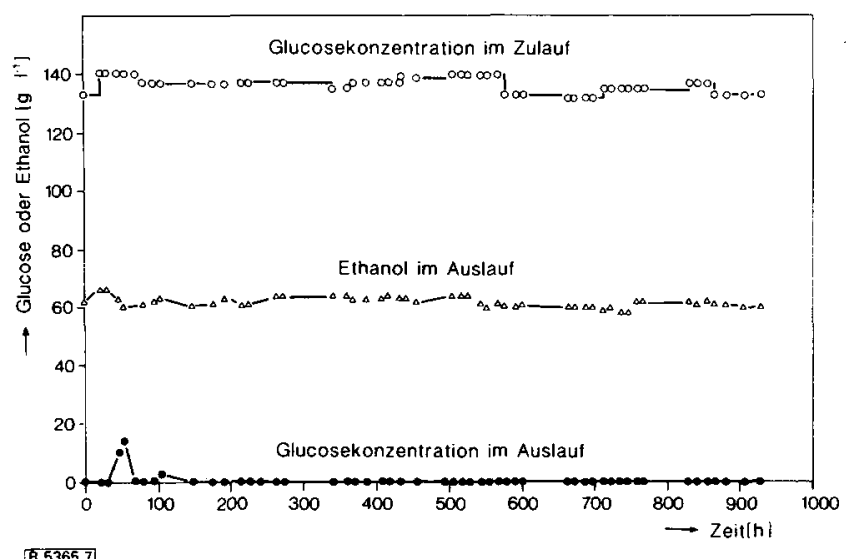

Abb. 7. Kontinuierliche Ethanol-Produktion mit Zymomonas mobilis bei einer Verdünnungsrate von $0,07 \mathrm{~h}^{-1}$.

Da für eine wirtschaftliche Ethanol-Produktion die Kosten für den Zucker von sehr großer Bedeutung sind, wurde die Vergärung einer enzymatisch verzuckerten Abfallstärke mit Zymomonas mobilis näher untersucht [11]. Kürzlich wurde bei der Firma Pfeifer \& Langen ein Verfahren zur Gewinnung von Glukosesirup aus Weizenmehl in Betrieb genommen (Abb. 8). Hierbei wird zunächst das Weizenmehl in eine reine A-Stärkefraktion, eine Proteinfraktion und in eine Rückstandsfraktion separiert. Die reine A-Stärkefraktion wird anschließend mit Hilfe von Enzymen zu einem Flüssigzuckerprodukt umgesetzt, das in der Lebensmittelindustrie Verwendung findet. In den USA werden zur Zeit jährlich etwa 2,5 Mio. Tonnen Maisstärke mit Hilfe von Amylasen und Amyloglucosidasen zu Flüssigzucker umgewandelt. Die Rückstandsfraktion enthält neben Stärke noch Faserstoffe, Proteine und Lipide, so daß diese Fraktion für die Erzeugung von reinem Glukosesirup nicht geeignet ist. Es konnte gezeigt werden, daß nach enzymatischer Verflüssigung und Verzuckerung der Abfallstärke die Glukose von Zymomonas mobilis sehr effizient zu Ethanol vergoren werden kann. Bei einer Glukose-Konzentration von $120 \mathrm{~g} / \mathrm{l}$ wurden in kontinuierlicher Kultur bei Verdünnungsraten von 0,06 bis $0,08 \mathrm{~h}^{-1} 99 \%$ der zugegebenen Glukose umgesetzt, die Ethanol-Produktivität lag bei 3,5 bis $4,5 \mathrm{~g} / \mathrm{l} \mathrm{h}$.

Diese Daten haben dazu geführt, daß Pfeifer \& Langen 1984 eine Anlage für die kontinuierliche Ethanol-Produktion mit Zymomonas mobilis aus dieser Abfall-Stärkefraktion errichtet hat (Abb. 9). In zwei $70 \mathrm{~m}^{3}$ Fermentern können täglich etwa $10000196 \%$ igen Alkohols produziert werden. Die Inte- 


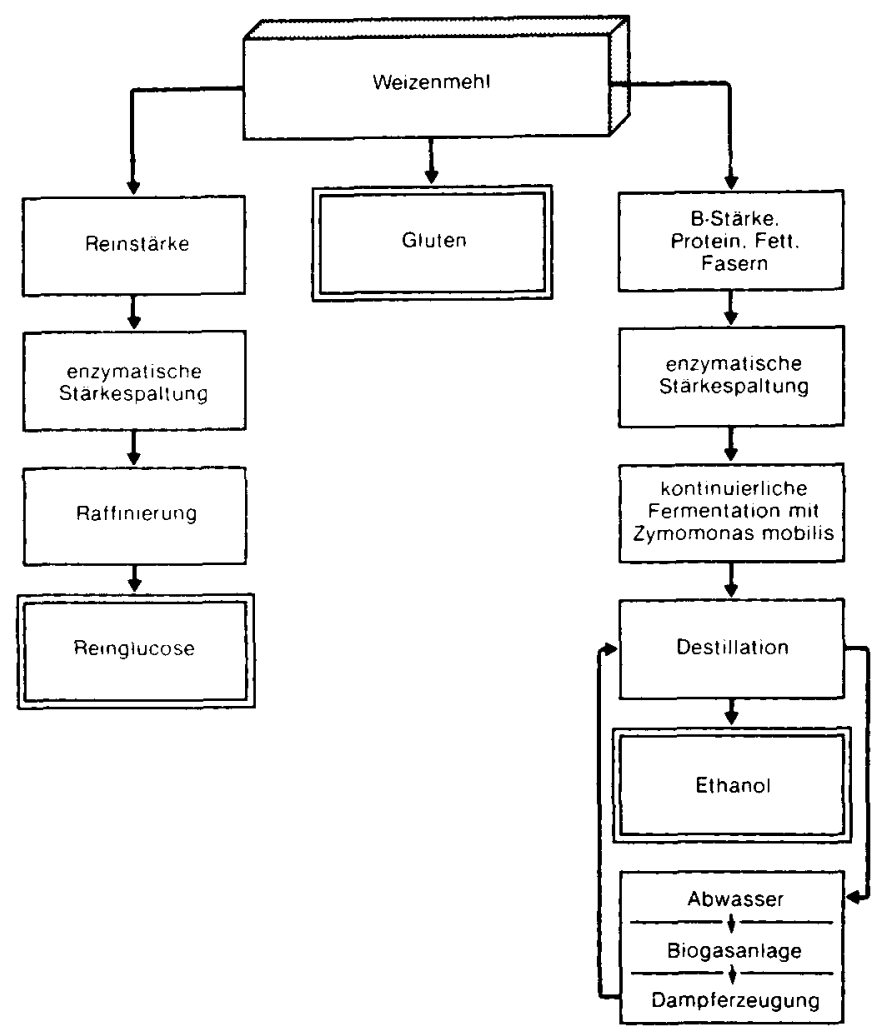

Abb. 8. Produktion von Glucose, Gluten und Ethanol aus Weizenmehl.

gration dieser Ethanol-Fermentation in den Gesamtprozeß der Weizenmehl-Verarbeitung ist in Abb. 8 dargestellt. Hieraus ist zu ersehen, daß nach der Vergärung der Glukose zu Ethanol und nach dessen Abdestillation ein Abwasser (Schlempe) anfällt, welches neben der Bakterienbiomasse noch Fasern, Proteine und Fette enthält, die Zymomonas mobilis nicht verwerten konnte. Um auch diese organischen Reststoffe noch sinnvoll verwerten zu können, wird das Schlempe-Abwasser in eine Biogasanlage gegeben, in der eine Mischkultur aus verschiedenen anaeroben Bakterien die organischen Verbindungen weitgehend zu Biogas, einem Gemisch aus Methan und $\mathrm{CO}_{2}$, abbaut. Das hierbei erzeugte $\mathrm{Me}-$ than wird zum Heizen der Destillationsapparatur genutzt.

\section{Ausblick}

Während die Hefen und Zymomonas mobilis Ethanol aus Glukose, Fruktose und Saccharose mit Ausbeuten von 90 bis $95 \%$ bezogen auf den theoretisch maximal erreichbaren Wert bilden, gibł es bis heute weder Hefen noch Bakterienstämme, die in der Lage sind, mit ähnlich hohen Ausbeuten Pentosen, wie z. B. Xylose oder Arabinose, zu Ethanol zu vergären. Da bei der Ethanol-Fermentation die Kosten für das Substrat bis zu 70\% der Gesamtkosten ausmachen, könnte die Wirtschaftlichkeit der Ethanol-Herstellung ganz wesentlich dadurch verbessert werden, daß nicht nur die Hexosen sondern auch die Pentosen der pflanzlichen Biomasse zu Ethanol umgesetzt werden. Es gibt eine Reihe von fakultativ und obligat anaeroben Bakterien, die in der Lage sind, auch Pentosen zu vergären. Alle diese Organismen bilden jedoch aus dem Zucker, neben Ethanol und $\mathrm{CO}_{2}$, noch erhebliche Mengen anderer Gärungsprodukte, wie z.B. Essigsäure, Ameisensäure, Milchsäure, Aceton und Wasserstoff. So produziert z. B. das fakultativ anaerobe Bakterium Bacillus macerans aus Xylose in Abhängigkeit von der Sauerstoff-Konzentration neben

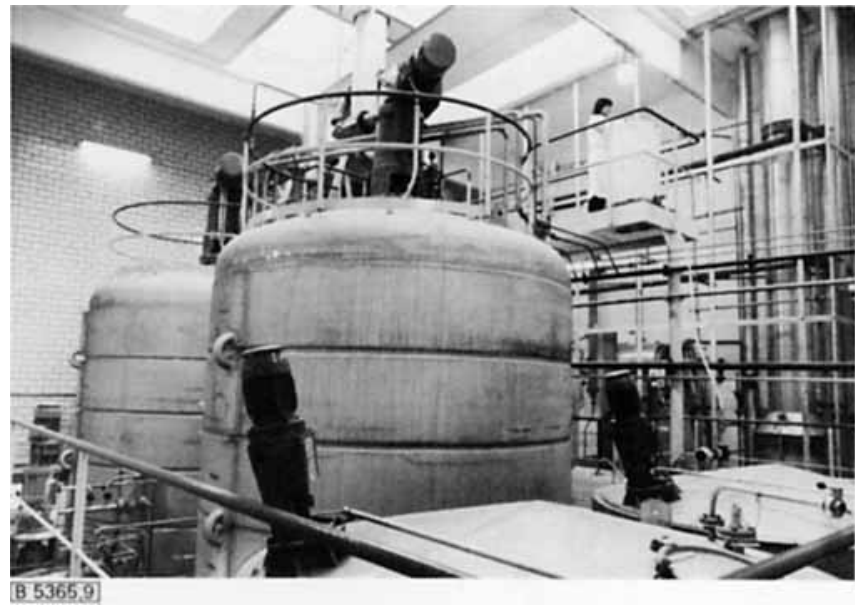

Abb. 9. Zwei Fermenter $\left(\right.$ je $70 \mathrm{~m}^{3}$ ) für die industrielle Ethanolproduktion mit Zymomonas mobilis bei der Firma Pfeifer \& Langen, Dormagen.

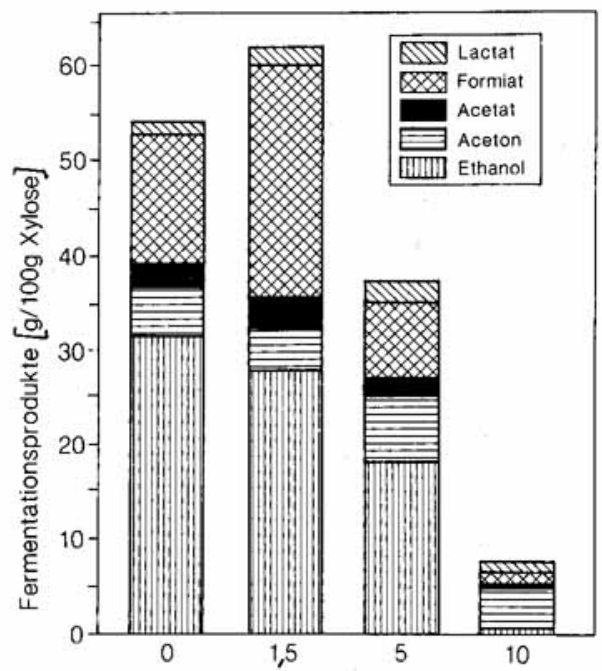

Sauerstoff-Konzentration [\%Luftsättigung] B 5365.10

Abb. 10. Einfluß der Sauerstoffkonzentration auf die Produktbildung bei der Xylose-Vergärung durch Bacillus macerans.

Ethanol noch unterschiedliche Mengen an Ameisensäure, Essigsäure, Milchsäure und Aceton [12] (Abb. 10). Wie stoffwechselphysiologische Untersuchungen gezeigt haben, werden die Pentosen in den Bakterien über den Pentosephosphat-Weg und den Glycolyse-Weg zu Pyruvat umgesetzt (Abb. 11). Im Gegensatz zu Zymomonas mobilis fehlt aber diesen Bakterien ein wichtiges Schlüsselenzym für die Ethanol-Gärung, die Pyruvat-Decarboxylase, welche Pyruvat zu Acetaldehyd und Kohlendioxid umsetzt. In den fakultativ anaeroben Bakterien wird das Pyruvat mit Hilfe einer Pyruvat-Formiat-Lyase zu Ameisensäure und Acetyl-CoA umgewandelt, während die strikt anaeroben Bakterien z. B. Clostridien eine Pyruvat-Ferredoxin-Oxidoreduktase und Hydrogenase besitzen, die Pyruvat zu Acetyl-CoA, Wasserstoff und $\mathrm{CO}_{2}$ umsetzen. Durch weitere enzymatische Reaktionen kann dann das Acetyl-CoA teilweise zu Ethanol reduziert werden. Milchsäure entsteht aus Pyruvat mit Hilfe der LactatDehydrogenase, die in Bakterien häufig durch Fructose-1,6diphosphat oder Fructose-6-phosphat aktiviert wird. Da den bislang untersuchten Pentose vergärenden Bakterien die Pyruvat-Decarboxylase fehlt und sie deshalb neben Ethanol und $\mathrm{CO}_{2}$ erhebliche Mengen anderer Gärungsprodukte bilden, sollte eine Übertragung des Gens für die Pyruvat- 


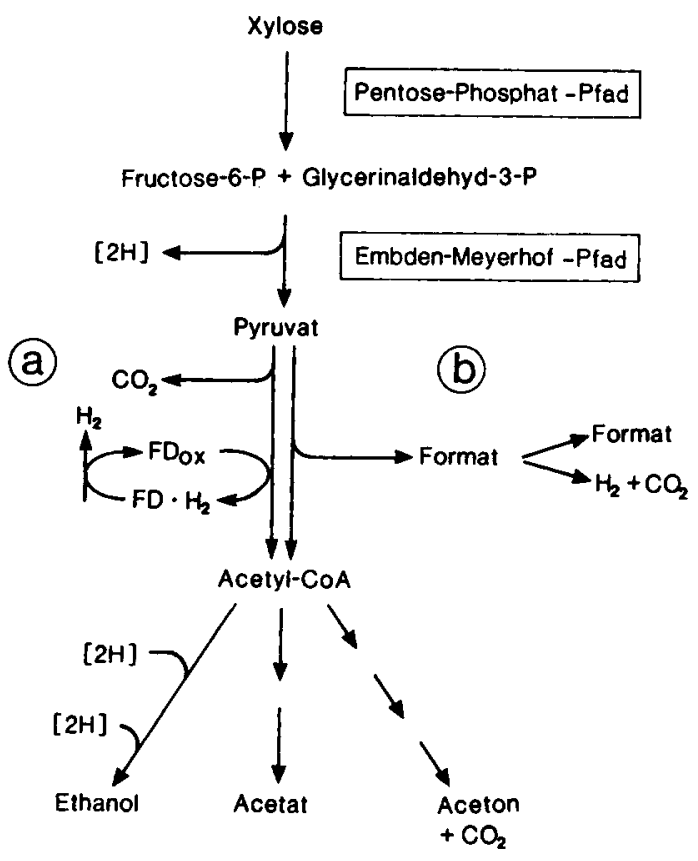

Abb. 11. Bakterielle Stoffwechselwege zur Vergärung von Pentosen. a) Pyruvat-Ferredoxin-Oxidoreduktase; b) Pyruvat-FormiatLyase.

\section{Tabelle 3.}

Gärungsprodukte (in g Produkt/g Zucker) von Escherichia coli und Klebsiella planticola, Wildtyp- und genetisch-modifizierten Stämmen, die das Gen für die Pyruvat-Decarboxylase aus Zymomonas mobilis enthalten. Die theoretisch maximale Ethanol-Ausbeute ist $0,51 \mathrm{~g} / \mathrm{g}$ Zucker.

\begin{tabular}{lcccc}
\hline & $\begin{array}{c}\text { Klebsiella planticola [15] } \\
\text { Wildtyp Transkonjugante }\end{array}$ & $\begin{array}{c}\text { Escherichia coli [14] } \\
\text { Wildtyp Transkonjugante }\end{array}$ \\
\hline Ethanol & 0,22 & 0,40 & 0,12 & 0,42 \\
Acetat & 0,25 & 0,06 & 0,19 & 0,01 \\
Lactat & 0,03 & 0 & 0,23 & 0,01 \\
Succinat & n.b. ${ }^{1)}$ & n.b. & 0,02 & 0,01 \\
Formiat & 0 & 0 & 0,02 & 0 \\
2,3-Butandiol & 0,02 & 0 & n.b. & n.b. \\
\hline
\end{tabular}

1) n.b.: nicht bestimmt.

Decarboxylase aus Zymomonas mobilis diese Organismen auch zu "reinen Ethanol-Gärern" machen. Erste Experimente zur Ubertragung dieses Gens in Escherichia coli und in Klebsiella planticola zeigen, daß die so veränderten Stämme bei der Vergärung von Zucker in der Tat wesentlich weniger organische Säuren im Vergleich zu dem Wildtyp bilden [13-15] (Tab. 3). Das Zwischenprodukt Pyruvat wird in diesen Stämmen anscheinend weitestgehend mit der neu „eingepflanzten" Pyruvat-Decarboxylase zu Acetaldehyd und dann weiter zu Ethanol umgesetzt. Es bleibt somit nur wenig Pyruvat für die Bildung von Essigsäure, Ameisensäure oder Milchsäure übrig. Diese ersten Ergebnisse weisen darauf hin, daß es auf diesem Weg möglich ist, Bakterien auch für eine effiziente Vergärung von Pentosen zu Ethanol zu entwickeln.

Eingegangen am 31. März 1987 [B 5365]

\section{Literatur}

[1] Giesel, H.: Nachr. Chem. Tech. Lab. 32 (1984) S. 316/325.

[2] Rogers, P. L.; Lee, K. J.; Skotnicki, M. L.; Tribe, D. E.: Adv. Biochem. Eng. 23 (1982) S. 37/84.

[3] Rogers, P. L.; Goodman, A. E.; Heyes, R. H.: Microbiol. Sci. 1 (1984) S. 133/136.

[4] Swings, J.; De Ley, J.: Bacteriol. Rev. 41 (1977) S. 1/46.

[5] Belaich, J. P.; Senez, J. C.: J. Bacteriol. 89 (1965) S. 1195/ 1200.

[6] Bringer, S.; Finn, R. K.; Sahm, H.: Arch. Microbiol. 139 (1984) S. $376 / 381$.

[7] Ingram, L. O.: Tibtech, February 1986, S. 40/44

[8] Ourisson, G.; Albrecht, P.; Rohmer, M.: Spektrum der Wissenschaft, Oktober (1984), S. 54/64.

[9] Bringer, S.; Härtner, T., Poralla, K.; Sahm, H.: Arch. Microbiol. 140 (1985) S. 312/316.

[10] Schmidt, A.; Bringer-Meyer, S.; Poralla, K.; Sahm, H.: Appl. Microbiol. Biotechnol. 25 (1986) S. 32/36.

[11] Bringer, S.; Sahm, H.; Zwyzen, W.: Biotechnol. Bioeng. Symp. 14 (1984) S. 311/319.

[12] Schepers, H. J.; Bringer-Meyer, S.; Sahm, H.: Z. Naturforsch. $42 c$ (1987) S. 401/417.

[13] Bringer-Meyer, S.;Schimz, K.-L.; Sahm, H.: Arch. Microbiol. 146 (1986) S. 105/110.

[14] Bräu, B.; Sahm, H.: Arch. Microbiol. 144 (1986) S. 296/301.

[15] Tolan, J. S.; Finn, R. K.: Appl. Environ. Microbiol. (1987), im Druck.

[16] Finn, R. K.; Bringer, S.; Sahm, H.: Appl. Microbiol. Biotechnol. 19 (1984) S. 161/166.

[17] Bringer, S.; Durst, E.; Sahm, H.; Finn, R. K.: Biotechnol. Bioeng. Symp. 14 (1984) S. 269/278.

[18] Murray, W. D.; Khan, A. W.; van den Berg, L.: Int. J. Syst. Bacteriol. 32 (1982) S. 132/135.

[19] Carreira, L. H.; Ljungdahl, L. G., in: Liquid fuel developments (Wise, D. L. Hrsg.), CRC Press Inc., Boca Raton, FL, 1983, S. $1 / 29$.

[20] Schmid, U.; Giesel, H.; Schoberth, S. M.; Sahm, H.: System. Appl. Microbiol. 8 (1986) S. 80/85. 